\title{
Screening Method
}

National Cancer Institute

\section{Source}

National Cancer Institute. Screening Method. NCI Thesaurus. Code C67056.

Any method of detecting the presence of disease. 